\title{
Radiative $b$-hadron decays at LHCb
}

\author{
Carlos Sánchez Mayordomo* $\dagger$ \\ Instituto de Física Corpuscular (Universidad de Valencia - CSIC), Spain \\ E-mail: carlos.sanchez.mayordomodcern.ch
}

Radiative decays of $b$-hadrons are ideal to search for New Physics effects. These decays are sensitive to the polarization of the emitted photon, which can help to constrain New Physics models in a complementary way to the branching fraction measurements. In this work, I present several results by the $\mathrm{LHCb}$ collaboration related to the photon polarization in $b \rightarrow s \gamma$ transitions, with special emphasis put on the new time-dependent $B_{s}^{0} \rightarrow \phi \gamma$ analysis. Other analyses discussed are the observation of a non-zero polarization in $B^{+} \rightarrow K^{+} \pi^{-} \pi^{+} \gamma$ decays, and the angular analysis of $B^{0} \rightarrow K^{* 0} e^{+} e^{-}$at the photon pole.

38th International Conference on High Energy Physics

3-10 August 2016

Chicago, USA

\footnotetext{
*Speaker.

${ }^{\dagger}$ On behalf of the LHCb collaboration.
} 


\section{Introduction}

Radiative $b$-hadron decays involve $b \rightarrow(s, d) \gamma$ transitions. These flavour changing neutral current (FCNC) processes are only allowed at loop level in the Standard Model (SM) and, therefore, are highly sensitive to New Physics (NP) effects, since new virtual particles can enter in the loop and modify the decay rate or the helicity structure.

Since the weak force only couples to left-handed quarks, the emitted photon in $b \rightarrow(s, d) \gamma$ is predominantly left-handed in the SM. However, in many extensions of the SM the right-handed contribution is enhanced, making the photon polarization a sensitive test for new phenomena [1].

The effective Hamiltonian describing the $b \rightarrow s$ transition is given by:

$$
H_{\mathrm{eff}}=-\frac{4 G_{F}}{\sqrt{2}} V_{\mathrm{tb}} V_{\mathrm{ts}}^{*} \sum_{i}\left(C_{i} O_{i}+C_{i}^{\prime} O_{i}^{\prime}\right)+\text { h.c. }
$$

where the Wilson coefficients $C_{i}^{\left({ }^{\prime}\right)}$ contain information on the short-distance physics, and describe the strength in which a given local operator $O_{i}^{\left({ }^{\prime}\right)}$ enters in the Hamiltonian. Primed operators have the chirality flipped, with very supressed coefficients in the SM. The radiative penguin is sensitive to the so-called electromagnetic operator:

$$
O_{7}^{\left({ }^{\prime}\right)}=\frac{e}{16 \pi^{2}} m_{b}\left(\bar{s} \sigma_{\mu v} P_{R(L)} b\right) F^{\mu v}
$$

where $P_{R, L}=\left(1 \pm \gamma_{5}\right) / 2$. The photon polarization can be expressed in terms of the electromagnetic Wilson coefficients:

$$
\lambda_{\gamma}=\frac{\left|C_{7}^{\prime}\right|^{2}-\left|C_{7}\right|^{2}}{\left|C_{7}^{\prime}\right|^{2}+\left|C_{7}\right|^{2}}
$$

Hence, measuring the photon polarization allows to constrain $C_{7}^{\left({ }^{\prime}\right)}$, which is supressed in the SM by the ratio of the light quark over the heavy quark $C_{7}^{\prime} / C_{7}=m_{s} / m_{b} \simeq 0.02$.

In this document, I review three analyses by the LHCb collaboration related to the photon polarization. In 2013, the LHCb published the first observation of the photon polarization in $b \rightarrow s \gamma$ transitions using $B^{+} \rightarrow K^{+} \pi^{-} \pi^{+} \gamma$ decays [2]. Two years later, an angular analysis of the $B^{0} \rightarrow K^{* 0} e^{+} e^{-}$decay in the low $q^{2}$ region was published, measuring observables sensitive to the $C_{7}^{\left({ }^{\prime}\right)}$ Wilson coefficients [3]. A new analysis on the time-dependent $B_{s}^{0} \rightarrow \phi \gamma$ decay rate has been performed by LHCb, measuring the mass-eigenstate rate asymmetry $A^{\Delta}$ for the first time [4]. All the discussed analyses are performed using the complete Run I dataset collected by LHCb, of $p p$-collisions at $\sqrt{s}=7 \mathrm{TeV}$ in 2011 and $\sqrt{s}=8 \mathrm{TeV}$ in 2012, corresponding to $3 \mathrm{fb}^{-1}$.

\section{First observation of the photon polarization in $B^{+} \rightarrow K^{+} \pi^{-} \pi^{+} \gamma$}

The LHCb collaboration observed for the first time the photon polarization in radiative $b$-hadron decays, using $B^{+} \rightarrow K^{+} \pi^{-} \pi^{+} \gamma$ data [2]. An angular analysis of the photon direction with respect to the $K^{+} \pi^{-} \pi^{+}$plane in their center-of-mass frame allows to distinguish between a polarized and a non-polarized photon through the measurement of the up-down asymmetry, which is proportional to the photon polarization $\lambda_{\gamma}[5,6]$. 
The up-down asymmetry is measured in four regions of the $K^{+} \pi^{-} \pi^{+}$invariant mass sensitive to different resonant contributions. The significance of all the bins combined is 5.2 standard deviations, confirming that the photon is polarized. However, the current limited knowledge of the $K^{+} \pi^{-} \pi^{+}$resonant system prevents the translation of this result into an actual value of the photon polarization parameter $\lambda_{\gamma}$.
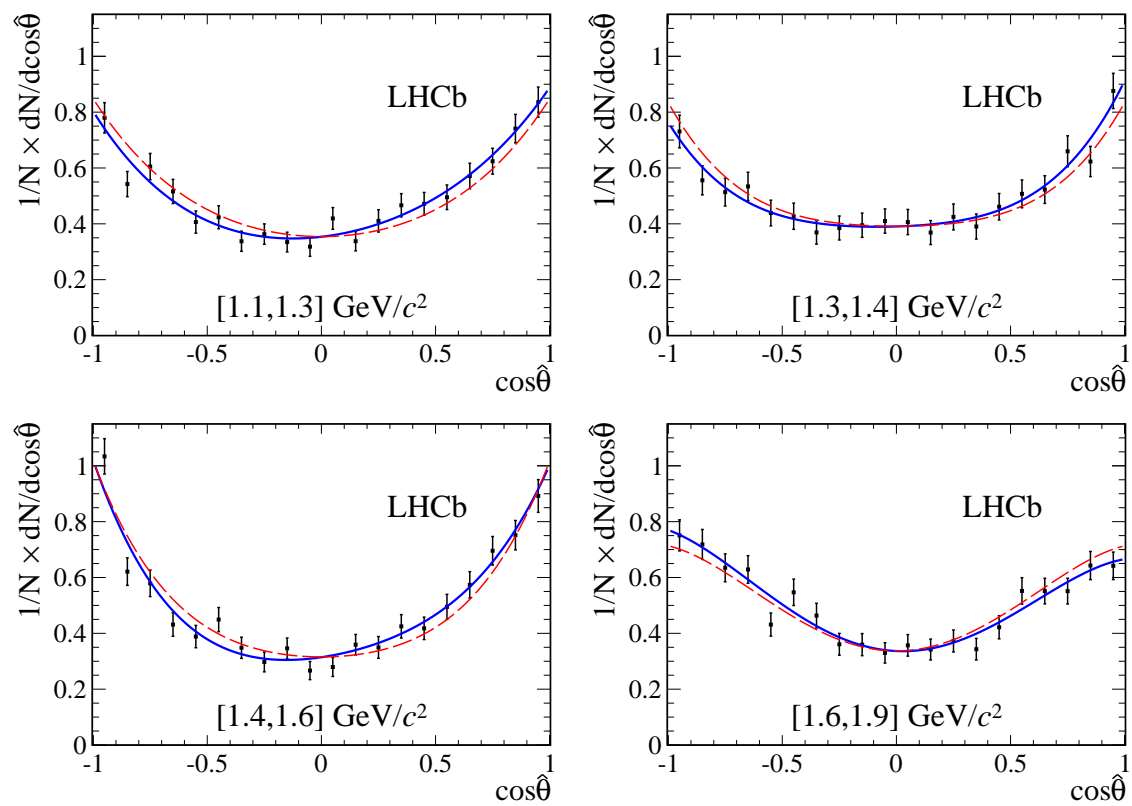

Figure 1: Photon angular distributions in four $K^{+} \pi^{-} \pi^{+}$invariant mass regions. In blue, the best fit. In red, the hypothesis of zero polarization.

\section{Angular analysis of $B^{0} \rightarrow K^{* 0} e^{+} e^{-}$decays at the photon pole}

The photon polarization information can be extracted from the angular analysis of the $B^{0} \rightarrow$ $K^{* 0} e^{+} e^{-}$decay in the low dielectron invariant mass region $\left(q^{2}\right)[7,8]$. In general, this decay can be mediated by electroweak box and penguin diagrams in the SM, but the virtual photon contribution dominates in the low $-q^{2}$ region, making it sensitive to the photon polarization. The observables $A_{\mathrm{T}}^{(2)}$ and $A_{\mathrm{T}}^{\mathrm{Im}}$ appearing in the differential decay rate are sensitive to the Wilson coefficients $C_{7}$ and $C_{7}^{\prime}$ in the limit $q^{2} \rightarrow 0$ [8]:

$$
A_{\mathrm{T}}^{(2)}\left(q^{2} \rightarrow 0\right)=\frac{2 \operatorname{Re}\left(C_{7} C_{7}^{\prime}\right)}{\left|C_{7}\right|^{2}+\left|C_{7}^{\prime}\right|^{2}}, \quad A_{\mathrm{T}}^{\operatorname{Im}}\left(q^{2} \rightarrow 0\right)=\frac{2 \operatorname{Im}\left(C_{7} C_{7}^{\prime}\right)}{\left|C_{7}\right|^{2}+\left|C_{7}^{\prime}\right|^{2}},
$$

depending on the real and imaginary parts, respectively.

These observables have been measured by the LHCb collaboration in the $q^{2}$ range between 0.002 and $1.120 \mathrm{GeV}^{2} / c^{4}$ (in which the Equations 3.1 are inaccurate by only 5\%) [3]:

$$
A_{\mathrm{T}}^{(2)}=-0.23 \pm 0.23 \pm 0.05, \quad A_{\mathrm{T}}^{\mathrm{Im}}=+0.14 \pm 0.22 \pm 0.05 .
$$

These values are consistent with the SM prediction, which are very close to zero. Currently, this measurement provides one of the strongest New Physics constraints on the electromagnetic Wilson coefficients. 


\section{Time-dependent $B_{s}^{0} \rightarrow \phi \gamma$ analysis}

The photon polarization can also be measured indirectly through time-dependent analysis of radiative $b$-hadron decays, since some observables are sensitive to the interference of the different helicity amplitudes [9]. Without identifying the flavour of the initial meson, the time-dependent decay rate of $B_{s}^{0} \rightarrow \phi \gamma$ decays can be written as:

$$
\Gamma_{B_{s}+\bar{B}_{s}}(t) \propto e^{-\Gamma_{s} t}\left[\cosh \left(\Delta \Gamma_{s} t / 2\right)-A^{\Delta} \sinh \left(\Delta \Gamma_{s} t / 2\right)\right],
$$

where the parameter $A^{\Delta}=\sin 2 \psi \cos \phi$ is related to the ratio of left- and right-handed amplitudes $\tan \psi=\left|A_{L}\right| /\left|A_{R}\right|$, and $\phi=\phi_{s}-\phi_{L}-\phi_{R}$. In the SM, $\phi=0$ and $A_{\mathrm{SM}}^{\Delta}=0.047_{-0.025}^{+0.029}$ [9]. This observable can also be written in terms of the Wilson coefficients:

$$
A^{\Delta} \simeq \frac{2 \operatorname{Re}\left(e^{-i \phi_{s}} C_{7} C_{7}^{\prime}\right)}{\left|C_{7}\right|^{2}+\left|C_{7}^{\prime}\right|^{2}}
$$

The LHCb experiment measured for the first time this parameter from a sample of $4200 B_{s}^{0} \rightarrow$ $\phi\left(\rightarrow K^{+} K^{-}\right) \gamma$ signal events [4]. The $B^{0} \rightarrow K^{* 0}\left(\rightarrow K^{ \pm} \pi^{\mp}\right) \gamma$ decay is used as a control channel since the branching ratio is six times larger and the decay rate is not affected by New Physics. Signal and background yields are extracted from the mass distribution, as shown in Figure 2.
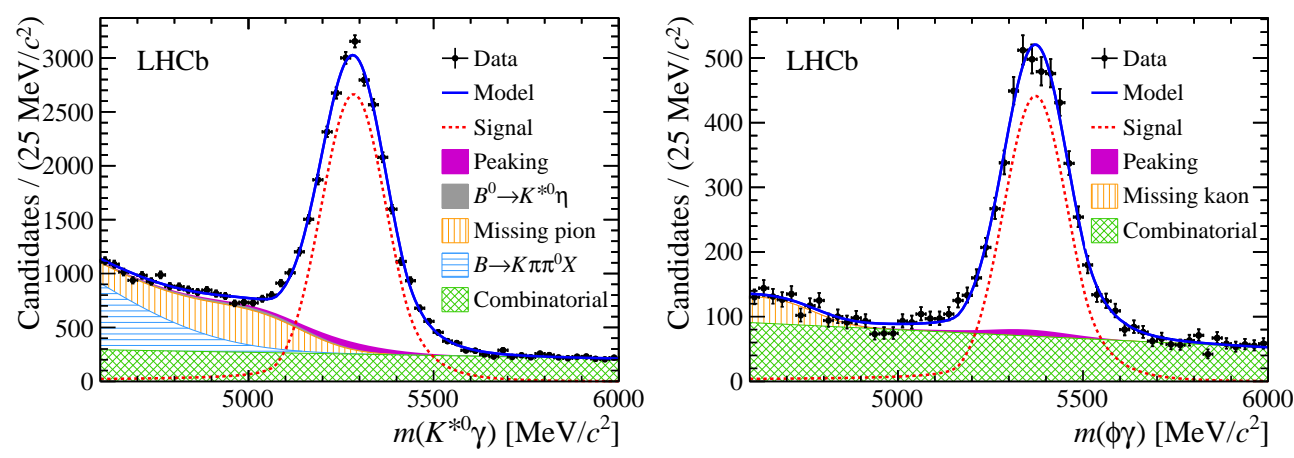

Figure 2: Mass distribution of the initial meson for $B_{s}^{0} \rightarrow \phi \gamma$ (left) and $B^{0} \rightarrow K^{* 0} \gamma$ (right). For the signal, a double-tailed Crystal Ball function is used. The combinatorial background is characterized by a first-order polynominal, and the partially reconstructed backgrounds are described by an ARGUS convolved with a Gaussian.

The distribution of the background-subtracted decay time of the initial meson is fitted simultaneously in the $B_{s}^{0} \rightarrow \phi \gamma$ and $B^{0} \rightarrow K^{* 0} \gamma$ samples, using an unbinned maximum likelihood fit (Figure 3 left). The physical decay time is multiplied by the detector acceptance, and then convolved with a resolution function. The acceptance is extracted from simulation, and validated with $B^{0} \rightarrow K^{* 0} \gamma$ data by fitting the lifetime $\tau_{B_{d}}^{\mathrm{fit}}=1.524 \pm 0.013 \mathrm{ps}$, which is compatible with the World Average [10]. A binned fit to the ratio of $B_{s}^{0} \rightarrow \phi \gamma$ over $B^{0} \rightarrow K^{* 0} \gamma$ yields was also developed (Figure 3 right), with compatible results.

The obtained value for $A^{\Delta}[4]$ :

$$
A^{\Delta}=-0.988_{-0.52}^{+0.46} \text { (stat.) }{ }_{-0.20}^{+0.23} \text { (syst.) }
$$



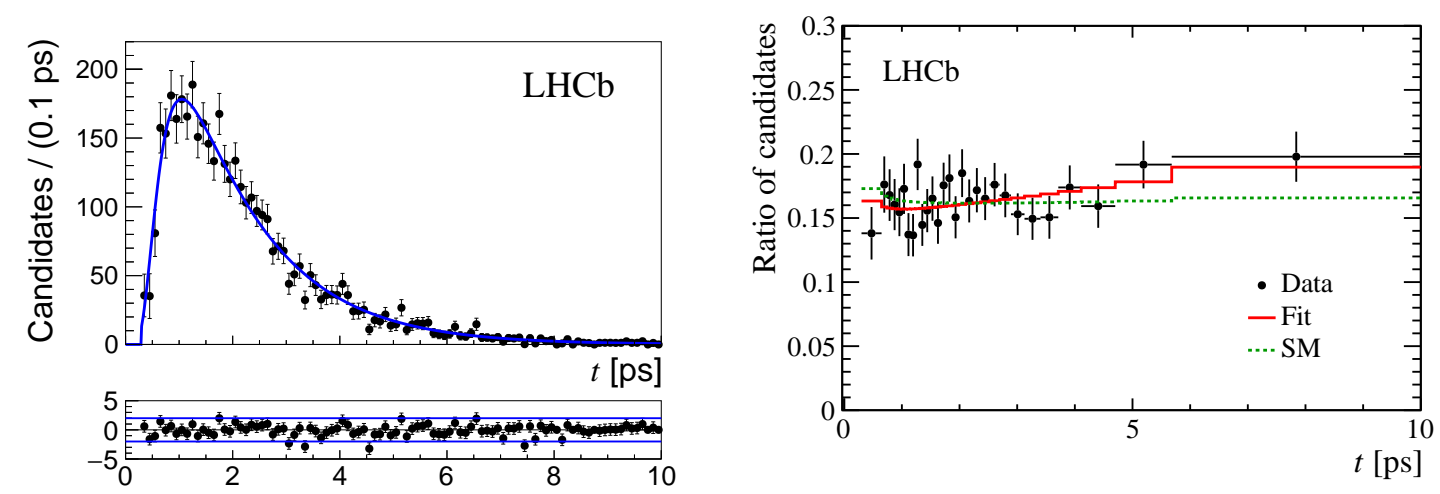

Figure 3: Left, the $B_{s}^{0} \rightarrow \phi \gamma$ proper time distribution, with the unbinned fit superimposed in blue. Right, the ratio of $B_{s}^{0} \rightarrow \phi \gamma$ and $B^{0} \rightarrow K^{* 0} \gamma$ yields, with the binned fit in red and the SM expectation in green.

is consistent with the SM expectation within two standard deviations. This is the first measurement of an observable sensitive to the photon polarization in $B_{s}^{0}$ decays.

The measurement is dominated by the statistical uncertainty, and will be reduced adding Run II data. The main systematic contribution is coming from the background evaluation, specifically the background mass modelling and the correlation of the mass with the decay time. This will be improved in the future with a comprehensive study of the background sources.

\section{Constraints on New Physics}

Radiative $b$-hadron decays are ideal to constrain the $C_{7}^{\left({ }^{(}\right)}$Wilson coefficients, since they are unaffected by $C_{9}^{\left({ }^{\prime}\right)}$ or $C_{10}^{\left({ }^{\prime}\right)}$ [11, 12]. Figure 4 shows the constraints on the real part of $C_{7}$ and $C_{7}^{\prime}$ (left) and in the $C_{7}^{\prime}$ complex plane (right) from radiative measurements, using the flavio software [13]. The SM contribution has been subtracted in the plotted quantities, and the non-plotted ones are assumed to be SM. One standard deviation contours are shown for each contribution, with the two standard deviation region also drawn for the combination.

$C_{7}$ is constrained mainly by the branching ratios, dominated by the inclusive $B \rightarrow X_{s} \gamma$. The dominant constraint to $C_{7}^{\prime}$ comes from the $B^{0} \rightarrow K^{* 0} e^{+} e^{-}$angular analysis, which is sensitive to the real and imaginary parts. The new $A^{\Delta}$ measurement constrains $\operatorname{Re} C_{7}^{\prime}$ and shows a preference for a non-zero value, but is still consistent with the SM. The combination of all the radiative measurements is as well compatible with the SM prediction within one standard deviation.

\section{References}

[1] Atwood D. et al., Mixing-induced CP asymmetries in radiative B decays in and beyond the Standard Model, Phys. Rev. Lett. 79185 [hep-ph/9704272].

[2] Aaij R. et al. (LHCb collaboration), Observation of photon polarization in the $b \rightarrow s \gamma$ transition, Phys. Rev. Lett. 112161801 [hep-ex/1402.6852].

[3] Aaij R. et al. (LHCb collaboration), Angular analysis of the $B^{0} \rightarrow K^{* 0} e^{+} e^{-}$decay in the low- $q^{2}$ region, JHEP 04 (2015) 064 [hep-ex/1501.03038]. 

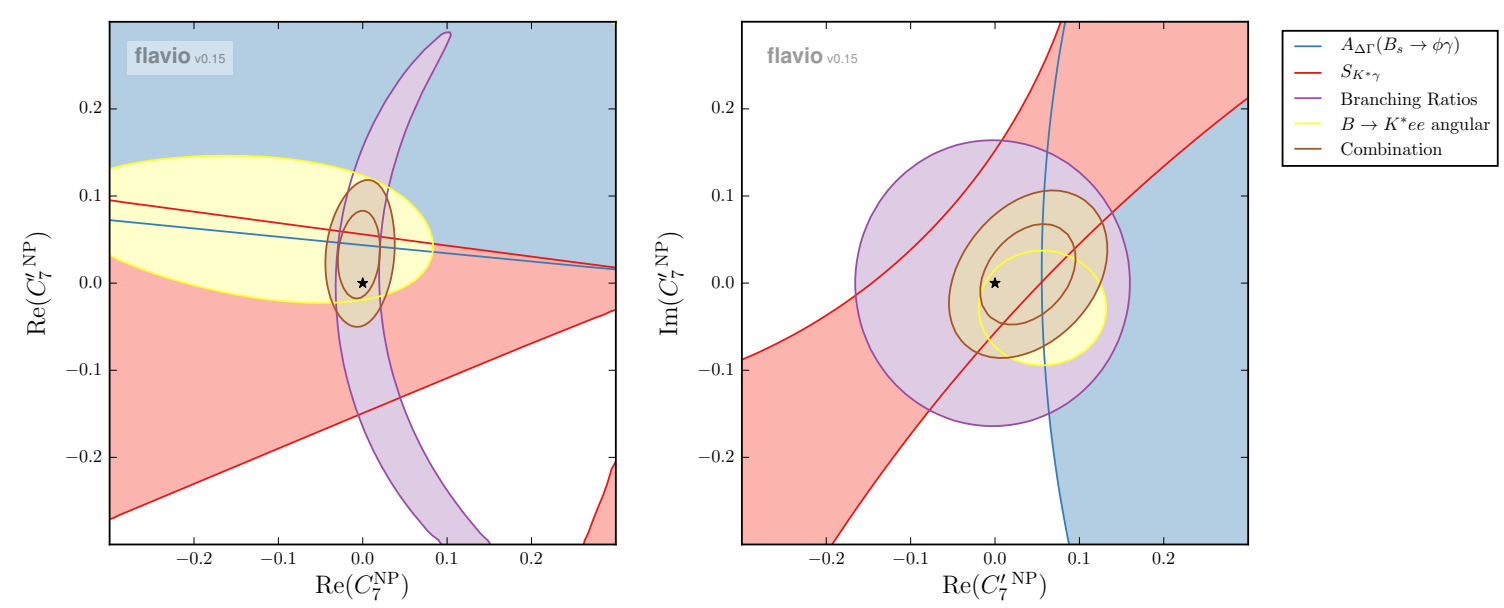

Figure 4: Constraints on the Wilson coefficients $C_{7}$ and $C_{7}^{\prime}$. Left, on the real part of the coefficients. Right, on the $C_{7}^{\prime}$ complex plane. The combination, in brown, is consistent with the SM prediction (star).

[4] Aaij R. et al. (LHCb collaboration), First experimental study of photon polarization in radiative $B_{s}^{0}$ decays [hep-ex/1609.02032].

[5] Gronau M. and Pirjol D. Photon polarization in radiative B decays Phys. Rev. D 66 (2002) 054008 [hep-ph/0205065].

[6] Kou E. et al., Determining the photon polarization of the $b \rightarrow s \gamma$ using the $B \rightarrow K_{1}(1270) \gamma \rightarrow(K \pi \pi) \gamma$ decay, Phys. Rev. D 83 (2011) 094007 [hep-ph/1011.6593].

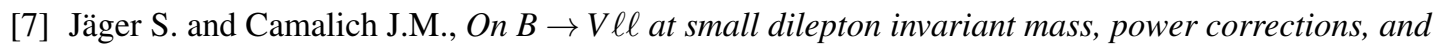
new physics, JHEP 05 (2013) 043 [hep-ph/1212.2263].

[8] Becirevic D. and Schneider E. On transverse asymmetries in $B \rightarrow K^{*} \ell^{+} \ell^{-}$Nucl. Phys. B 8542 (2012) 321-339 [hep-ph/1106.3283].

[9] Muheim F. et al., Exploiting the width difference in $B_{s}^{0} \rightarrow \phi \gamma$, Phys. Lett. B664 (2008) 174-179 [hep-ph/0802.0876].

[10] Amhis, Y. et al. (Heavy Flavour Averaging Group), Averages of b-hadron, c-hadron, and $\tau$-lepton properties as of summer 2014, [hep-ex/1412.7515], and online update at http://www.slac.stanford.edu/xorg/hfag

[11] Descotes-Genon S. et al., Exploring New Physics in the C7-C7' plane, JHEP 06 (2011) 099 [hep-ph/1104.3342].

[12] Paul A. and Straub D.M., Constraints on new physics from radiative B decays, [hep-ph/1608.02556].

[13] Straub D.M. et al., flavio v0.11.1, Zenodo (2016) [doi:10.5281/ zenodo.59955], and update at https://github.com/flav-io/flavio 